David Conde-Estévez ${ }^{1,2}$ Melisa Barrantes-González ${ }^{3}$ Maria Renne Cotrina Soliz ${ }^{4}$ Santiago Grau ${ }^{1,3}$

\title{
Successful management of remdesivir extravasation
}

\author{
${ }^{1}$ Infectious Disease Control. Department of Pharmacy. Hospital Universitari del Mar. Passeig Maritim 25-29, E-08003, \\ Barcelona, Spain \\ ${ }^{2}$ Hospital del Mar Medical Research Institute (IMIM), Hospital Universitari del Mar, Barcelona, Spain \\ ${ }^{3}$ Hospital Clínic, Barcelona, Spain \\ ${ }^{4}$ Hospital Universitari del Mar., Barcelona, Spain
}

Article history

Received: 10 October 2021; Accepted: 26 November 2021; Published: 4 February 2022

\section{Sir,}

Extravasation is a potentially severe complication of treatments by intravenous administration. Their consequences may include tissue necrosis, compartment syndrome, infection, ulceration, and partial or total loss of limb function $[1,2]$. Information about extravasation management is scarce and often limited to case reports. Primarily, it was described remdesivir as a vesicant drug due to formation of blisters, hemorrhage, and localized edema when extravasated [3]. Recently three cases of remdesivir extravasation were described, two of them treated with hyaluronidase injections [4]. However suitable early management of remdesivir extravasation remains unknown. Here, a successful management case of remdesivir extravasation with only dry heat is first described.

A 68-year-old woman was admitted at hospital with sore cough, dyspnoea and dysgeusia. Initially, she presented with typical pulmonary infiltrates. However, on day 6 after admission, the patient's clinical status deteriorated rapidly and diagnosed with severe SARS-CoV-2 infection PCR positive. So, dexamethasone and five days remdesivir treatment was initiated. Initially, treatment was overall well tolerated. The fourth course of remdesivir was given by peripheral intravenous injection into the radial vein at the left wrist using an infusion pump. Remdesivir $100 \mathrm{mg}$ in $250 \mathrm{~mL}$ (concentration of $0.4 \mathrm{mg} /$ $\mathrm{mL}$ ) was infused within $120 \mathrm{~min}(2.08 \mathrm{ml} / \mathrm{min})$. Fifteen minutes before completing remdesivir infusion, the patient complained of moderate pain around the site of injection. It was suspected an extravasation. On clinical examination, the patient had an approximately $4 \times 6 \mathrm{~cm}$ swollen area without erythema (see Figure 1A). General unspecific measures to treat extravasations were taken immediately as per our protocol: infusion was stopped and nurse aspired $5 \mathrm{ml}$ approximately, removed the needle, elevated the limb and called reference physician. According to acidic nature of remdesivir, it was recommended warm applications (dry warm compresses) for 15 minutes, every 8 hours, for 48 hours. Several medical controls were scheduled, and after 2 days after implementing these measures, patient recovered without complications (figure 1B). After ten days of follow-up, no sequel was observed, and patient was happily discharged home.

As far as we concerned, this is the first reported case of remdesivir extravasation with successful management in the literature treated with only dry warm. As described recently, hyaluronidase could be also an option after infiltration of acidic drugs to disperse and dilute the infiltrated drug (specially high amount of drugs) $[1,4]$. However, local injections of hyaluronidase can cause side effects such as local pruritus and allergic reactions [5].

Remdesivir has been authorized for emergency use in patients with severe SARS-CoV-2 infection as it reduced the median time to recovery from COVID-19 in a randomized controlled trial [6]. After a systematic review of published literature about remdesivir extravasations, no matched was obtained. General guidelines of non-cytotoxic extravasations recommend general measures as described before. Remdesivir was slightly acid ( $\mathrm{pH}=4.11)$. Acid exposure commonly leads to cellular desiccation, coagulative necrosis, and eschar formation. Edema, vasoconstriction, sloughing, and ulceration are common manifestations of acid-induced tissue injury. Management of acidic infiltrations remains supportive. Elevation, warm compresses, and attempts to remove the extravasated material are common nonpharmacologic treatment approaches [7].

The use of local warming therapy (dry heat) is based on the theory that it enhances vasodilation, thus enhancing the dispersion of the vesicant agent and decreasing drug accumulation in the local tissue. The use of local warming is recommended for the extravasation of non-DNA-binding vesicants.

The correct knowledge of remdesivir management, includ- 

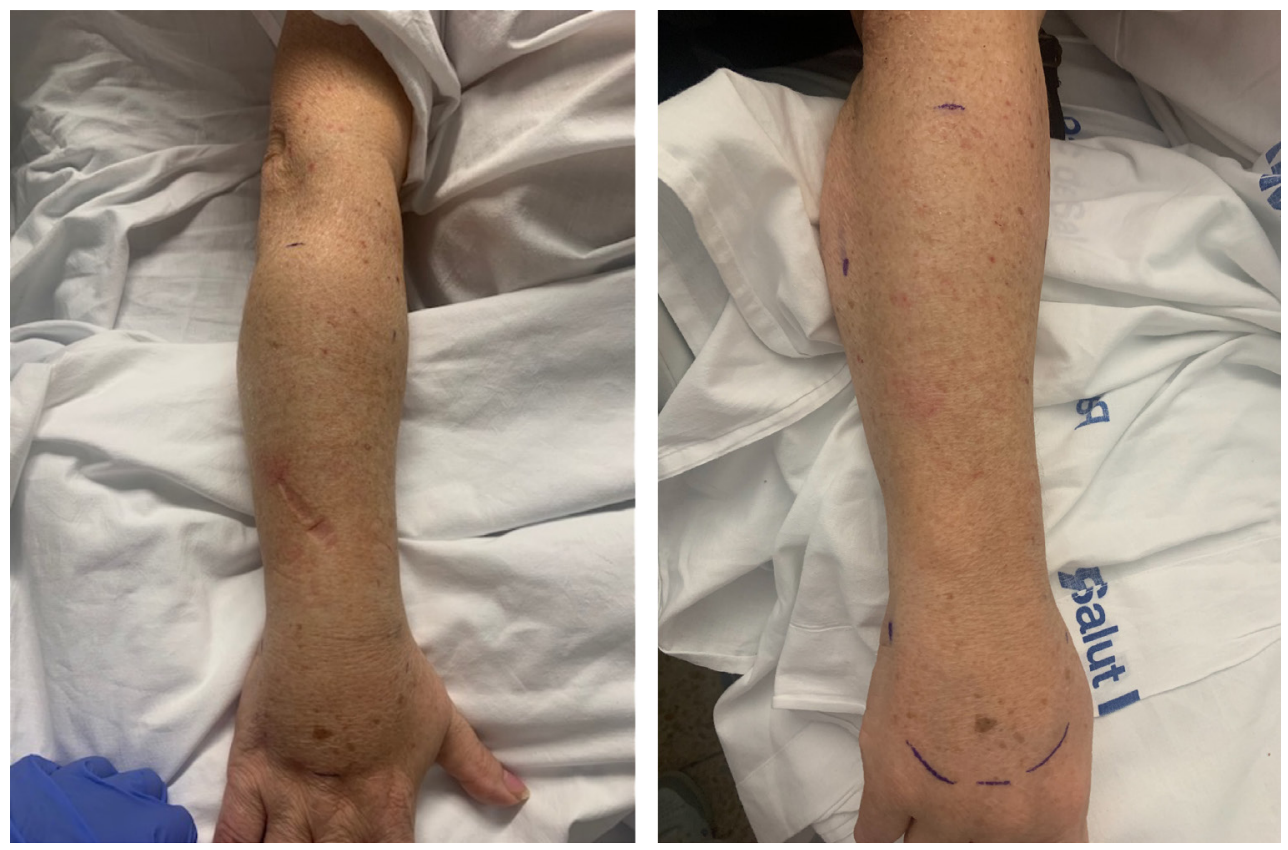

\begin{tabular}{l|l} 
Figure 1 & Macroscopic aspect of the lesion the day $0(A)$ and day $+2(B)$.
\end{tabular}

ing that of possible extravasations is essential since, despite its controversial use, the drugs available for COVID-19 disease are very limited. According to our case report, remdesivir extravasation may be managed effectively with only dry heat and general conservative measures.

\section{FUNDING}

None to declare

\section{CONFLICT OF INTEREST}

The authors declare no conflicts of interest.

\section{REFERENCES}

1 David V, Christou N, Etienne P, Almeida M, Roux A, Taibi A, Mathonnet M. Extravasation of Noncytotoxic Drugs. Ann. Pharmacother. 2020;54(8):804-14. doi;10.1177/1060028020903406.

2 Fidalgo JAP, Fabregat $L G$, Cervantes $A$, Margulies A, Vidall C, Roila et al. Management of Chemotherapy Extravasation: ESMO-EONS Clinical Practice Guidelines + on Behalf of the ESMO Guidelines Working Group *. ESMO Updat. Clin. Pract. Guidel. 2012;23:vii167vii173. doi;10.1093/annonc/mds294.

3 Kumar N, Kumar A, Pradhan S, Kumar A, Singh K. Painful Blisters of Left Hand Following Extravasation of Remdesivir Infusion in COVID-19. Indian J. Crit. Care Med. 2021;25(2):240-241. doi;10.5005/ JP-JOURNALS-10071-23732.
4 van Merendonk LN, Leeuwerik AF, den Brok MWJ, Hekking PW, Korevaar DA, Jacobs $\mathrm{CJ}$ et al. Peripheral Infiltration of Remdesivir in 3 Patients with COVID-19: Case Series and Discussion. Am J Health Syst Pharm. 2021. doi;10.1093/AJHP/ZXAB197.

5 Jung $H$. Hyaluronidase: An Overview of Its Properties Applications and Side Effects. Arch Plast Surg. 2020;47(4):297-300. doi;10.5999/APS.2020.00752.

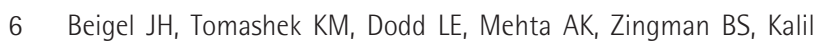
$A C$, et al. Remdesivir for the Treatment of Covid-19 - Final Report. N Engl J Med. 2020;383(19):1813-1826. doi;10.1056/nejmoa2007764.

7 Reynolds PM, Maclaren R, Mueller SW, Fish DN, Kiser TH. Management of Extravasation Injuries: A Focused Evaluation of Noncytotoxic Medications. Pharmacotherapy 2014;34(6):617-632. doi;10.1002/phar.1396. 